\title{
Separating the Chaff from the Wheat: on the Possible Origins of the Oblique Effect
}

\author{
Matthias S. Keil and Gabriel Cristóbal \\ Instituto de Óptica (Consejo Superior de Investigaciones Científicas), Serrano 121, 28006 Madrid \\ (Spain)
}

\begin{abstract}
The oblique effect refers to a better perception of horizontal and vertical image features as compared to oblique angles. This effect can be observed both in animals and humans. Recent neurophysiological data suggest that the basis of this effect lies in the structure of the primary visual cortex, where more cortical area is devoted to processing contours with angles at horizontal and vertical orientations (cardinal orientations). It has been suggested that this cortical feature has developed according to the statistical properties of natural scenes. To examine this hypothesis more in detail, we established six image classes, and categorized the images with respect to their semantical contents. From the images the oriented energy was calculated by using the corresponding power spectra. We defined simple measures for the degree (COR, cardinal vs. oblique energy ratio) and the skewness or anisotropy (AER, aligned energy ratio) of the alignment of energy at horizontal and vertical orientations. Our results provide evidence that (i) alignment depends highly on the environment, (ii) the degree of alignment drops off characteristically at higher frequencies, (iii) in natural images there is on the average an anisotropy in the distribution of energy at the cardinal orientations (i.e. a difference between the amount of vertical energy and horizontal energy). In the light of our results we further discuss whether the observed cortical anisotropy has its origin in phylogeny or ontogeny.
\end{abstract}




\section{Introduction}

Evolution can be seen as a process which stores and accumulates "knowledge" about the physical structure inherent in the environment genetically. These genes then generate by means of self-organizing chemical processes a phenotype with specific traits. Such traits can be of a more structural sort (e.g. design and shape of limbs, organs, ...) or might correspond to typical behavioral or motor patterns. However, these traits must have increased the fitness of the predecessors of an organism under consideration in order to be passed to the offspring. If we focus now on mammalian visual systems, we can see that different animals share common characteristics. For instance, the structure of classical receptive fields of cortical simple cells can be described by Gabor functions ${ }^{1}$. Another example is the oblique effect. This refers to enhanced perception (in the foveal retina) along the horizontal and vertical retinal axes of vision compared to contours at oblique angles. Hereafter we refer to these as cardinal axes. The effect reveals itself for instance in an orientation-dependent contrast sensitivity function or in an increased acuity to discriminate differences in orientation if they are close to the cardinal axes ${ }^{2}$. Again, the oblique effect can be observed in many different species, e.g. rats, ferrets, cats, monkeys, and humans ${ }^{3}$, although those animals see the world from different viewing angles. This implies that the physical structure of the environment provides constraints for the evolutionary process, and it is this structure that also exerts strong influences on the post-natal development of an organism. We now can ask if certain structures of the brain correspond to specific boundary-conditions for the evolutionary process, and if this turns out to be true, then we can expect to find them by analyzing the physical structure of the environment. An interesting experiment which provides strong support for proceeding in this way was done by Olshausen and Field ${ }^{4}$. They obtained localized, oriented and bandpass receptive fields which resemble the classical receptive fields of simple cells in the primary visual cortex by training an unsupervised learning algorithm with natural images. The network was designed to optimize two constraints. The first one was an average sparse activation of the network-units, and the second one was that 
the input must be recoverable from the output. This amounts to maximization of sparseness and preservation of information, respectively.

Apart from the evolutionary process, which is analogous to learning on a slow (phylogenetic) time scale, there exists a more flexible learning-scheme on an ontogenetic (faster) time scale. For example, if kittens are reared in cylinders painted with stripes of only a single orientation, simple cells will adapt by shifting their preferred orientation to the experienced one ${ }^{5}$. Thus, another important question refers to the time scale of development, i.e. whether we are faced with a phylogenetic or ontogenetic acquired trait. Consider again the rearing of kittens in a striped cylinder. If we assume that the preferred orientation of simple cells redistribute according to the experienced orientations during growing-up, then behavior might influence the outcome. Thus, head-rotation may broaden the distribution of cells away from the experienced orientation (but this seems not to be the case, $\sec ^{5}$ ).

Another example, in which one can assume a link between the physical structure of the environment and a structural trait of the mammalian visual system is the oblique effect. Let us first look at the brain. By considering the brain of ferrets, Chapman and Bonheoffer ${ }^{6}$ found, in accordance with the study of Coppola et al. ${ }^{7}$, that in all ferrets more cortical area is devoted to the processing of the cardinal axes of the corresponding foveal region. The size of this asymmetry showed some variation across the animals used in their experiments. Further, Coppola et al. ${ }^{7}$ reported that on the average about $2 \%$ more area of the primary visual cortex preferred horizontal to vertical orientations. Hereafter we will use the term anisotropy to denote this property. Our results for the natural image set are consistent with this neurophysiological finding for higher spatial frequencies.

Schall et al. ${ }^{8}$ compared the orientation of dendritic fields of retinal ganglion cells with their corresponding targets in cats. Again there was found an over-representation of the cardinal orientations in both retina and visual cortex. Note that although the visual systems of cats and ferrets are similar in their structure, they show a different developmental state at the time of birth. The cat visual system is about two or three weeks ahead in maturity ${ }^{9,10}$. Now we can look for corresponding statistical regularities in the physical structure of the 
environment. First indications were provided by Switkes et al. ${ }^{11}$. They calculated the power spectra of natural images by using optical Fourier analysis. Three classes were established, according to the landscapes or environments in which their images were taken. These comprised indoor carpentered scenes (19 pictures), outdoor carpentered scenes (23), and pastoral scenes (15). Under the assumption that visual experience alone can account for the oblique effect, they concluded that "since the implicit assumption that carpentered environments present more high frequency horizontal and vertical components than do non-carpentered environments is not supported by the data reported here, we may conclude either that environment has no effect in producing anisotropy, or that if it does, it is by a mechanism which is not specific for spatial frequency.". A later study, nevertheless, came up with different results $^{12}$. In this study Coppola et al. gathered photographs by means of adjusting their camera with a carpenter's level. This was done to achieve correct alignment with the horizon line. Again, images were categorized, in sets for indoor, outdoor, and natural scenes. From each class 50 images were selected randomly for further analysis. Images were not mentioned to be calibrated in order to compensate possible artifacts produced by the optical system of the camera. Orientation was determined by using $9 \times 9$ Sobel filters. This corresponds to the extraction of high spatial frequency content. A decreasing ratio of Sobel-magnitude at filter orientations $0^{\circ}$ and $180^{\circ}$, respectively, was observed in the picture categories indoor, outdoor and entirely natural. Interestingly, their results seem to be slightly different for $0^{\circ}$ and $180^{\circ}$ as well as for $90^{\circ}$ and $270^{\circ}$. The conclusions Coppola et al. drew give support to the hypothesis that the oblique effect has indeed its origin in the visual environment. However, two issues are not yet clarified. Besides of their selectivity for a certain orientation, simple cells also show a selectivity for spatial frequency. So, it is surprising that up to now no one has attempted to clarify the role of spatial frequency in the expression of the oblique effect, either neurophysiologically or statistically. But this requires a quantification of the oblique effect, and so we defined two measures, namely $C O R$ (cardinal vs. oblique energy ratio) and $A E R$ (aligned energy ratio). For instance, Coppola et al. ${ }^{12}$ discussed only the high frequency content of their images (they only briefly mentioned that their results also 
seem to hold for down-sampled versions of their images). Furthermore, they used aligned images, although we can not tacitly assume that a developing organism encounters a set of images stabilized in this way. In this sense the images we used were rather unbiased. It will be interesting to see whether on the average alignment is generated rather than introduced a priori. One further example is the study from van der Schaaf and van Hateren ${ }^{13}$. They discussed (although for a different image set than the one we were using here) the dependence of energy on orientation by using a power spectrum averaged over the image set and over spatial frequencies. No distinctions with respect to image classes were made. Another issue we will discuss here is whether the effect was produced during phylogeny or is established ontogenetically.

Methodical differences of our study from the described previous studies include the definition of five image classes. This allows for a more refined examination of visual environments. Furthermore, by defining two measures upon the oriented energy (OE) we are able to quantify the distribution of energy at cardinal vs. oblique angles as well as the anisotropy itself of the energy at the cardinal orientations. These measures are the cardinal vs. oblique energy ratio $(C O R)$ and the aligned energy ratio $(A E R)$.

To examine the frequency-dependencies of $C O R$ and $A E R$, we used localized band pass integration areas onto the two dimensional power (or energy) spectrum (ES). Inverse Fouriertransformation of these areas would yield Gabor-like filter pairs in quadrature phase in the spatial domain. It should be noted that this was not supposed to be an attempt to mimic the response-properties of cortical simple cells. Rather, we chose these methods to meet the well-known property of self-similarity of natural images ${ }^{14}$, and to obtain a good resolution in both angular and spatial-frequency dimensions. Stated differently, our concern here is to analyze exclusively the physical structure of the environment rather than to look at the world through the glasses of the mammalian visual system. We try to find the boundary-conditions or constraints which were exerted on the evolutionary process in order to understand its solutions.

Finally, we experimented with three different versions of the energy spectrum (ES) for cal- 
culating the oriented energy (OE) to demonstrate that the specific functional form which is used to read out the physical information is of no major importance for our results. We used, besides the unmodified (or direct) power spectrum a logarithmic and a $k^{2}$-windowed version (where $k$ is the spatial frequency).

\section{Image Properties and Classification}

Images were taken from the natural stimuli collection of the laboratory of Hans van Hateren $^{15}$. These images having each an original size of $1536 \times 1024$ pixels. They possess an angular resolution of approximatively 1 arcmin per pixel, which yields a maximum spatial frequency resolution of 30 cycles per degree (the human eye can resolve up to about 60 cycles per degree $\left.{ }^{16}\right)$. They were calibrated according to the procedure outlined in reference ${ }^{17}$. Therein can be found also a more detailed description of their properties.

As we proceeded with the calculation of the mean ES, we discovered an artifact common

to the images. It comes to light principally in the form of two broken vertical "lines" at high spatial frequencies (figure 2). This effect might have been generated through electrical interferences in the camera ${ }^{18}$, and can only be seen in the logarithmic ES (i.e. it is very small). We verified that this effect had no significant influence on our results.

We established six categories for the images, and a subset of van Hateren's image database was selected and classified accordingly. In what follows we will frequently make use of the contraction for each class, which will be denoted by italics.

The class city contains urban scenes. These show a strong prevalence of manufactured objects (e.g. houses, streets) compared to natural objects (like trees). If a photograph is taken of such scenes, one typically observes that the vertical edges of manufactured objects are parallel to the image frame. In other words, the horizon line (the line of gravity) in those images typically is parallel to the horizontal (vertical) bounds of the image. In this case we are speaking about (strong) alignment. The reason is that humans while taking such 
photographs in a straight direction of gaze usually tend to align their cameras. From this all images taken accordingly can considered to be well aligned, although we did not explicitly resort to, for example, a carpenter's level. Closer to nature are the scenes represented by the man. Here we find images containing relatively few manufactured objects compared to natural ones. Typically, in these scenes there can be seen a building hidden to some extent behind trees, or streets and fences embedded in a countryside. The frequency of manufactured objects is considerably less than in city. Like in city, the alignment in man is well in general. The images in the category natural contain no obvious manufactured objects. Instead we find in natural mainly scenes from inside the woods and then a few scenes from open landscapes with meadows, trees and seas. There are also scenes which were taken by photographing upward, and consequently this class contains some poorly aligned images as well. Compared to city or man we can consequently expect in natural a bigger variation of the alignment. With straight we established a category containing a selected subset of images of natural containing only scenes which were subjectively well aligned. The selected scenes in straight are further biased in the sense that they consist to a large number of scenes from inside the woods. In grass we find images that mainly show grass or flowers. There were also included some macro-photographs of flowers in this class. Moreover, in grass we can find many pictures taken from upside rather from a perspective corresponding to a small animal. Consequently, grass consists of both well aligned images and some for which alignment cannot be defined (i.e. those that correspond to a straight view onto the ground). Finally, nine pictures showing pure sky were examined. Alignment in this case is not defined, because these images show only one or a few clouds.

The number of pictures in each class were 121 for city, 188 for man, 241 for natural, 56 for straight, 49 for grass and 9 for sky . 


\section{Statistical Measurements}

\section{A. Calculation of the Energy Spectra}

For all measurements the original image size of 1024 rows times 1536 columns was reduced to a square image consisting of $1024 \times 1024$ pixels to perform an optimal fast Fourier transform. The resizing was made such that the first and the last 256 columns were dropped. Subsequently, each image was normalized to one and weighted by a Gaussian with $\sigma=1024 / 4 \sqrt{2}$ centered in the middle of the image. In this way the window-effect of the Fourier-transform is weakened. The windowed image was subsequently transformed into the spatial frequency domain and the ES was calculated as the square of the amplitude spectrum. The first row and first column were dropped to achieve correct centering. In this representation all energy values $E(\vec{k})$ corresponding to the same spatial frequency $k=\|\vec{k}\|$ are lying on a circle with radius $k$. Then the average two dimensional ES over all images in a category was calculated. Rather than relying on only the original ES we experimented both with a logarithmic and an "compensated" ES. The latter was calculated by multiplying the ES by $k^{2}$ isotropically (i.e. a circular window function). The motivation for doing so was the fact that the energy of natural images is approximately proportional to $1 / k^{2}$, see e.g. ${ }^{19,14}$. Thus, after applying this function, the energy can expected to be roughly independent of spatial frequency (i.e. constant along the line of radius).

\section{B. Calculation of Oriented Energy: The "Club-Integration-Scheme" (CIS)}

From here on we operate in the spatial frequency domain. The oriented energy (OE) was calculated by using what we have named the "Club-Integration-Scheme" (CIS), because the integration area at a certain angle of orientation resembles a club, as can be seen in figure 1a. The total integration area covered is shown in figure $1 \mathrm{~b}$. The clubs were shaped both in angular and in radial direction by using the power of a cosine, i.e. $\cos ^{2 \alpha} \Theta$. Here $\Theta$ refers to the phase angles at a reference orientation plus the angle of rotation $\phi$ of the club. In 
radial direction, which corresponds to the frequency bandwidth, $\alpha \equiv \alpha_{k}=4$ was chosen. In angular direction, which corresponds to the orientation selectivity, $\alpha \equiv \alpha_{\Theta}=400$ was chosen, which makes the club rather slim. The peak value of the cosine was specified to be at a frequency of approximatively $k=18$ cycles per degree. In this way the intermediate frequencies exert a dominant influence on the results. Hence, the club served as a weighting scheme for the underlying energies. We also experimented with unweighted clubs (i.e. which simply cut out a piece of the ES) and the outcomes were similar. Furthermore, the results do not depend critically on either of the selected parameter-values. The integration scheme was compensated for small sampling artifacts. These occur because the integration area at oblique orientations is bigger than at angles corresponding to cardinal orientations due to small nonzero weights. The angular resolution was set to $\Delta \phi=5^{\circ}$. This yields in total 72 oriented energies $E_{\alpha}(\phi)$. To simplify notation, the index $\alpha$ refers to the specific settings of both $\alpha_{k}$ and $\alpha_{\Theta}$. By definition, ES has even symmetry, i.e. it holds that $E_{\alpha}(\phi)=$ $E_{\alpha}\left(\phi+180^{\circ}\right)$. By taking this into account we only need to analyze half of all orientations.

[Fig. 1 about here.]

\section{Measures on the Oriented Energy}

Let $\tilde{E}_{\alpha}^{(\sigma)}(\phi)$ be the Gaussian-smoothed, oriented energy (OE)

$$
\tilde{E}_{\alpha}^{(\sigma)}(\phi)=E_{\alpha}(\psi) \otimes \frac{1}{\sqrt{2 \pi} \sigma} e^{-\frac{(\phi-\psi)^{2}}{2 \sigma^{2}}}
$$

where the symbol " $\otimes$ " denotes convolution in angle $\psi$, and $\phi$ is the orientation on which the Gaussian is centered, and $\sigma$ is the Gaussian's standard deviation. By that we can define the energy at cardinal orientations as

$$
E_{\text {cardinal }}=\tilde{E}_{\alpha}^{\left(\sigma_{c}\right)}\left(0^{o}\right)+\tilde{E}_{\alpha}^{\left(\sigma_{c}\right)}\left(90^{\circ}\right)
$$


and the energy at oblique orientations as

$$
E_{\text {oblique }}=\tilde{E}_{\alpha}^{\left(\sigma_{o}\right)}\left(45^{o}\right)+\tilde{E}_{\alpha}^{\left(\sigma_{o}\right)}\left(135^{o}\right) .
$$

The spread of the Gaussian at the oblique orientations $\sigma_{o}$ was chosen twice the standard deviation of the Gaussian at the cardinal orientations $\sigma_{c}$, hence $\sigma_{o}=2 \times \sigma_{c}$. By setting $\sigma_{c} \equiv \sigma$ we can drop the indices.

Then, the cardinal vs. oblique energy ratio $C O R$ is defined as

$$
\operatorname{COR}(\sigma)=\frac{E_{\text {cardinal }}-E_{\text {oblique }}}{E_{\text {cardinal }}+E_{\text {oblique }}}
$$

Smoothing (the convolution with the Gaussian) is applied because in this way information from adjoining orientations (i.e. adjacent to the angles corresponding to the centers of the Gaussians) is made available, although with less weight. As a further consequence the $C O R$ is also getting a more smooth or stable measure, respectively: the alignment is never perfectly equal in each considered image. This is due to the fact that the cardinal orientations in the image (i.e. line of horizon and line of gravity) are never perfectly aligned with the CIS at the corresponding orientations (i.e. $90^{\circ}$ and $0^{\circ}$, respectively). Thus we obtain some sort of fluctuations around the cardinal and oblique orientations from image to image, which in a certain sense can be considered as noise. By applying smoothing we can alleviate this effect to some extent.

The denominator provides a normalization, such that $-1 \leq C O R \leq+1$. A positive $C O R$ indicates that we found more energy at cardinal (i.e. horizontal and vertical) orientations, and this is a measure of the strength of the oblique effect. If a $C O R$-value is located around zero, then the energy can be expected to be distributed more or less uniformly over the considered orientations, and there will be no oblique effect. Similarly, if the $C O R$ is negative, 
then there will be more energy at oblique orientations rather than a oblique effect.

Now we have to specify the free parameter $\sigma$. For all calculations $\sigma=1.5$ was chosen, which gives a reasonable trade-off between robustness and blurring the OE. To estimate when the $C O R$ is significant, we calculated the mean $C O R(\sigma)$ for uniform distributed noise. For each value of $\sigma$, the corresponding $C O R(\sigma)$ was averaged over 100,000 trials (i.e. noise samples). In this way we get an average or mean value (which should ideally be zero) and an associated absolute deviation (we chose the absolute deviation rather than the standard deviation). The deviation defines a positive and a negative limit around zero, which yields a range in which our values do not possess significance (within this range we cannot discriminate the signal from noise). Consequently, if we lster obtain a $C O R$ which lies above or below this range $\Delta C O R_{\text {crit }}$, this must be due to a property of the data different from noise. Only in this situation we can consider the $C O R$ as significant, and we can be sure that the cardinal orientations indeed contain more energy than the oblique ones (or vice versa). For $\sigma=1.5$ the $C O R$ range is defined as $\Delta C O R_{\text {crit }}:=\{C O R \mid-0.089 \leq C O R \leq 0.089\}$.

We also experimented with different values of $\sigma$ and other $C O R$-definitions. The main effect in both cases was a shift of the considered $C O R$-values by an offset value. The shape of the $C O R$-plots (i.e. by the $C O R$ vs. spatial frequency) nearly remained similar.

To measure the anisotropy of the energy at cardinal orientations we define the aligned energy ratio, $A E R$, analogously to the $C O R$ :

$$
A E R(\sigma)=\frac{\tilde{E}_{\alpha}\left(0^{o}\right)-\tilde{E}_{\alpha}\left(90^{\circ}\right)}{\tilde{E}_{\alpha}\left(0^{\circ}\right)+\tilde{E}_{\alpha}\left(90^{\circ}\right)} .
$$

This measure gives the difference in energy corresponding to vertical $\left(0^{\circ}\right.$ club orientation) and horizontal ( $90^{\circ}$ club orientation) image features, and the denominator provides a normalization. Thus, it holds that $-1 \leq A E R \leq 1$. Negative values of the $A E R$ indicate 
a relative preponderance of horizontal image features in the range of frequencies under consideration. The opposite holds true for vertical image features, indicated by positive values. As above, $\sigma=1.5$ was chosen. This yields $\triangle A E R_{\text {crit }}:=\{A E R \mid-0.151 \leq A E R \leq 0.151\}$. To calculate the dependencies on spatial frequencies of $A E R$ and $C O R$, we set the $\alpha$-values to $\alpha_{\Theta}=\alpha_{k}=150$. This yields small, isotropic, blob-like integration-areas. The integration areas at different frequencies are simply rescaled versions of each other. The frequency interval $[8,508]$ cycles per image is passed through (or sampled) linearly with a resolution of $\Delta k=5$ cycles per image. As already mentioned in the introduction, the specific values for $\alpha_{\Theta}$ and $\alpha_{k}$, respectively, were chosen such that a good resolution is obtained, rather than to set them to values corresponding to the classical receptive fields of simple cells. For

example, if we used an octave scheme for sampling the frequency axis ${ }^{14}$, we would not get a sufficiently smooth plot which would allow us to see the frequency-dependence of the $C O R$ or $A E R$ with a good resolution along the spatial frequency axis.

\section{Results and Discussion}

The logarithmic averaged power spectra for each class can be seen in figure 2. A pseudo-color map was applied for purposes of visualization. Figure 2a shows the mean energy spectrum (ES) of city. There is a clearly visible strong concentration of energy along the cardinal axes, and the shape of the distribution resembles a diamond. Note that the influence of perspective can be seen best in this class. Perspective affects in the first place horizontal lines, which present different angles when projected onto a two dimensional plane. This leads to a broader distribution of horizontal image features, and for this reason energy is diffusing away from the vertical axis in the ES. The energy around the horizontal ES-axis (vertical image features) is clearly more concentrated. This can be better seen in figure 3a. Here the oriented energy (OE) is presented. The distribution around the orientation of $90^{\circ}$ (vertical club, see figure 1) corresponding to horizontal image features is again much broader. Nevertheless, all distributions here are very marked and show sharp peaks, due to a prevalence 
of "rectangular" objects (e.g. buildings) over natural ones. The dependence of the OE on spatial frequency can be seen in figure 4a. This plot suggest a nearly stable alignment over the considered frequency range, which is confirmed by considering the $C O R$-plot in figure 5a. The decrement of the degree of alignment at higher spatial frequencies at approximately 15 cycles per degree seems to be a characteristic property of the classes man, natural and straight. For the falling off can be observed in all classes, this might indicate that alignment in general gets weaker as the spatial frequency increases. This means that if we look at objects at a fine scale, we will see that the associated features (lines, edges) occur at other than the cardinal orientations with higher probability. In the $A E R$-plot for city (figure $5 \mathrm{~b}$ ), the energy is roughly distributed equally on the cardinal axes, although with a slight bias in favor of vertical image features, in the range of low spatial frequencies. A curiosity in the shape of a marked peak can be found in the OE-plot in figure 4a near the orientation of $90^{\circ}$ and at a frequency roughly around 17.5 cycles per degree (because of the three dimensional presentation this seems actually to be at around 20 cycles per degree). Interestingly, this peak is also present in man at the same orientation and at the same spatial frequency. Thus, because it is not present in any of the image sets which contain only natural images, there must be some typical manufactured feature which is shared by both sets city and man. Of course this is not easy to track down. Note that there is also another peculiar peak (but smaller) in figure $4 \mathrm{~b}$ in the $0^{\circ} / 180^{\circ}$ orientation in man at around 17 cycles per degree.

The ES of man (figure 2b) resembles again a diamond, but the energy at high spatial frequencies seems to be more poorly concentrated at the cardinal axes than in city. This indicates that features (lines, edges) corresponding to high spatial frequencies are distributed more uniformly over all orientations. Nevertheless, the ES of man shows once again distinct peaks in figure $3 \mathrm{~b}$, although the presence of natural features introduces now a broadening of the energy-distribution around both cardinal orientations. Furthermore, if we compare the ES of man and natural (figure $2 \mathrm{~b}$ and c, respectively), we observe that adding manufactured object(s) to a natural scene obviously increases the energy along the cardinal axes over 
the whole range of frequencies. The marked concentration of energy along the cardinal orientations in man along nearly the whole range of spatial frequencies (despite of a relative preponderance of natural objects over the manufactured ones) can verified in figure $4 \mathrm{~b}$ and 5 c. Interestingly, the $A E R$ plot (figure $5 \mathrm{~d}$ ) of man (as opposed to city) indicates now a significant probability of finding more vertically oriented image features in the range of low spatial frequencies.

The ES of natural is roughly circular (figure 2c), although with slight bumps, which are the remains of the corners of the diamond. One can, if at all, see a weak vertical line at lower frequencies in the ES. But inspection of OE in figure 3c clearly shows peaks, even though the energy is distributed very broadly around the orientation of $90^{\circ} / 270^{\circ}$. To understand this better, let us consider the frequency dependence of the $\mathrm{OE}$ in figure $4 \mathrm{c}$ as well as the $C O R$ (figure 5e, continuous line) and the $A E R$ (figure 5f, lower line). Obviously, we are faced with strong alignment at low spatial frequencies, which falls off by roughly $60 \%$ compared to its maximum value at high spatial frequencies. Another conspicuous feature is the sign reversal of the $A E R$ at around 15 cycles per degree. The consistent interpretation of this effect is that the low spatial frequencies are stronger influenced by vertical aligned objects, whereas at high spatial frequencies horizontal features predominate. Let us consider again figure 4c. Note that the image features with horizontal orientation have roughly the same energy over the whole range of frequencies, while vertical image features "lose" energy at the same time. Therefore, the sign-reversal of the $A E R$ is probably caused by a "disappearance" of vertically oriented image features at higher spatial frequencies. We cannot rule out that this may be a peculiarity of the scenes we used. A consistent explanation, however, would be that tree-trunks at short distances significantly influence the lower spatial frequencies. With increasing viewing distance in a scene the trunks are getting more and more "invisible". This is because they are getting covered by branches and trunks from trees situated in the foreground, and so the probability of seeing a tree-trunk which is far away is relatively low. At the same time, the branches can be considered to deliver the main contribution for the energy around the horizontal orientations. This holds, as suggested by figure 4c, over 
the whole range of frequencies. Moreover, because trunks and branches hide themselves mutually, additional spatial frequencies are generated. Although this might happen with equal probability at all orientations, it might in this case especially add additional energy to the horizontal orientations. However, this is speculation and should be verified explicitly. By all means it would be interesting to see whether this behavior of the $A E R$ (figure $5 \mathrm{f}$ ) is mirrored in the primary visual cortex, too. If this is the case, then we will find a relative bigger number of cells preferring vertical orientations to horizontal ones at low spatial frequencies, whereas the opposite holds true for high spatial frequencies. At intermediate frequencies (around 10 to 15 cycles per degree), the respective number of cells should be more or less equal. Furthermore, if we take into account that the human visual system can resolve up to 60 cycles per degree ${ }^{16}$, it would also be interesting to see whether the decrement of the $A E R$ with increasing frequency is continued up to 60 cycles per degree, or whether there is another inflection point beyond 30 cycles per degree, which is the maximum spatial frequency examined here.

Another source of energy at high spatial frequencies is grass, thickets or shrubbery, which is present to a certain extent in all images of man, natural, straight and grass. By inspecting figure $3 \mathrm{e}$ and figure $4 \mathrm{e}$ for grass we can see that grass, thickets and shrubbery also occur with some probability at vertical orientations, although there seem to be no preference for horizontal orientations, and the distribution around the orientation of $0^{\circ} / 180^{\circ}$ is relatively broad. This is quite surprising, because the images in grass are taken arbitrary relating to alignment. But despite of this, the relatively high degree of alignment, as suggested by the $C O R$-plot in figure $6 \mathrm{a}$, is caused exclusively by the non-existence of peaks at the horizontal orientation. This is reflected by a high value of the $A E R$ (near one) in figure $6 \mathrm{~b}$ and can also be seen in figure 4e. Moreover in figure 4e we see that the energy at low spatial frequencies is very noisy, because grass (thickets, shrubbery) contribute in the first place to high spatial frequencies. Note that in figure 2e the ES for grass shows characteristic rays emerging from the disk around the center. These fine rays correspond, of course, to some "misaligned" 
high-frequency features (i.e. at other than oblique orientations). This indicates that in grass the feature-orientations at high spatial frequencies are distributed more uniformly over the angular scale - a clue to poor alignment of the individual images and perhaps also to the small number of images (49) used in this class.

With straight is demonstrated what will happen if we pick out those images of natural which seem to be subjectively (i.e. to a human observer) well aligned. The principal difference to natural is an increase of the $0^{\circ} / 180^{\circ}$ peak relative to peak at $90^{\circ} / 270^{\circ}$ (figure $3 \mathrm{c}$ and $\mathrm{d}$, and figure $4 \mathrm{c}$ and $\mathrm{d}$ ). Note also that in figure $3 \mathrm{~d}$ we obtained a more noisy plot due to the smaller number of images in straight (241 in natural as against to 56 in straight ). In figure 5e the $C O R$ is presented for natural and straight in one plot. In figure $5 \mathrm{f}$ the $A E R$ is plotted for both classes. As can be easily seen, the main effect in both cases (i.e. $A E R$ and $C O R$ ) seems to be that the graphs for natural are shifted upward (i.e. to higher values of the $A E R$ and the $C O R$ ), while their shape seems to be maintained at the same time. This is a hint that, although natural contains some poorly aligned scenes, this in the end does not have significant effect. Thus, the effect of head-rotation on the overall statistics seems to be negligible, given that we consider the displacement of the $C O R$ as harmless in the sense that the relative number of cells at cardinal and oblique orientations is preserved. Moreover, the same seems to hold for the $A E R$. Here, the relative number of cells dedicated to the processing of horizontal and vertical orientations, seems to be preserved, too. However, to clarify whether head-rotation in any case amounts to merely a shift of $C O R$ and $A E R$, an additional study should be undertaken, which calculates the $C O R$ and $A E R$ for an image set as a function of mixing well and poorly aligned images, e.g. by systematically increasing the relative amount of rotated versions of the original images (if adequate camera-shots were not available).

In sky we examined only 9 images, so the results must be judged regarding this fact. As we can see in the ES of sky (figure 2f), the energy of such images is concentrated at 
lower spatial frequencies in an isotropic fashion. At high spatial frequencies only relatively small values of energy remain. For this reason the window-artifact of the numerical Fouriertransform becomes visible and leaves us with two spurious maxima in figure $3 \mathrm{f}$ at cardinal orientations. If we had a lot of images in sky, we ideally would obtain no dependency of the energy on orientation, as long as we would not have taken the images against the horizon. Single clouds above our heads normally do not possess a preferred orientation, whereas cloudy sky at the horizon present a strong horizontal component. However, our images only show single clouds as seen above our heads.

Despite of the window-artifact of the Fourier-transform, both the $C O R$ and the $A E R$ (figure $6 \mathrm{c}$ and $6 \mathrm{~d}$, respectively) yield more or less reliable results. The $C O R$ lies only in the noisy domain, that is to say it cannot be considered as significant. And in consideration of this, the $A E R$ does not possess any meaning.

The results in terms of $C O R$ and $A E R$ as calculated from the $\mathrm{OE}$ of figure 3 for each class are summarized in figure 7. In addition we demonstrated in these plots the effect of taking the logarithmic (circles) and the $k^{2}$-windowed (squares) ES, respectively, as input for the CIS. The differences between the different spectra are small, and more important, the monotony (in its mathematical sense) is preserved. Thus, if the visual system makes a monotonic transformation of the light-patterns impinging upon the retina, the statistical properties of those patterns should be conserved and should be reflected in the visual cortices.

We also calculated the $C O R$ s and $A E R$ for each individual image. These results are summarized in table 1 and 2, respectively. An extended version of this article including a discussion of the results for the individual images is available and can be requested from the authors.

[Fig. 2 about here.] 
[Fig. 3 about here.]

[Fig. 4 about here.]

[Fig. 5 about here.]

[Fig. 6 about here.]

[Fig. 7 about here.]

[Table 1 about here.]

[Table 2 about here.]

\section{Conclusions}

In this study we made a detailed examination of the alignment in natural scenes. As opposed to existing studies ${ }^{11,13,12}$, we quantified the degree of alignment $(C O R)$ and examined the dependence of this measures on spatial frequencies. Our results for high frequencies confirm quantitatively the outcomes of the study from Coppola et al. ${ }^{7}$, although our images were not manually aligned. This suggests that (i) the oblique effect can be seen in many kinds of natural images (city, man, natural) and in this sense natural images share common statistical structures which seem to be independent of the specific countryside in which they were gathered (Coppola et al. of course used images showing different landscapes), and (ii) alignment can be produced on the average up to some certain amount of counteracting head-movements. More importantly, we have seen that different sets of pictures generate alignment of different strength. With straight we have exemplarily shown how the alignment of natural changes if we only consider well aligned images. Further on, we quantified the anisotropy of the energy between the (cardinal) angles $\left(0^{\circ} / 180^{\circ}\right.$ vs. $\left.90^{\circ} / 270^{\circ}\right)$ by definition of the $A E R$. This enabled us to examine the frequency dependence of the anisotropy, captured by the behavior of the $A E R$. 
Again, for high frequencies we obtained quantitatively the same results as Coppola et al. ${ }^{7}$, and this again may be a hint at another generic property of natural images (see also figure 1(B) in the paper of van der Schaaf and van Hateren ${ }^{13}$ ). Remarkably, in natural we found a characteristic sign-reversal of the $A E R$ (figure $5 \mathrm{f}$ ), and its origin presumably lies in a covering scheme, i.e. objects located in the foreground partially occlude background objects (see the "results and discussion"-section).

To explain the higher probability of occurrence of features along the cardinal axes, Coppola et al. ${ }^{7}$ supposed that "the reason for a bias toward the cardinal axes in such different natural settings is presumably an omnipotent horizon dictated by the earth's surface (which guarantees horizontal components in most scenes) and an abundance of plants that use vertical support to counter the force of gravity and horizontal extension to capture sunlight with maximum efficiency.". However, in our natural image set those scenes showing open landscapes (i.e. exposing a horizon) are clearly in the minority (about $4 \%$ ), and thus the horizon cannot account for the energy at horizontal orientations in our results. Moreover, a straight line of horizon should on the contrary produce more narrow peaks at orientations of $90^{\circ} / 270^{\circ}$. This can be understood by considering the power-spectrum of a step function, which is typically sharply localized. The only candidates which remain in this range of spatial frequencies are branches of trees, on the grounds of the specific images we used.

Another remarkable finding is that there is a characteristic drop off with higher frequencies of the $C O R$, indicating that alignment gets less stable with increasing scale. It can be seen quite obvious in the classes city, man, natural and straight. This behavior of the $C O R$ can not be caused by the approximately $1 / k^{2}$ proportionality of the energy found in natural images ${ }^{14}$. We ruled this out by considering not only the original ES, but also the $k^{2}$-windowed ES, which caused a shift in the absolute values of the $C O R$ (and also the $A E R$ ), but failed to induce critically changes in the overall distribution. The reason for the falling off cannot be due to perspective. In city we have a preponderance of horizontal and 
vertical aligned features (lines, edges) due to manufactured objects. In this case perspective may be one source that the alignment is getting less stable with increasing frequencies. But perspective only affects the horizontal orientation, and equally contributes to all frequencies. Furthermore, perspective leads to a diffusion of energy away from the horizontal orientation. Consequently, the $A E R$ should indicate a relative preponderance of vertical image features. However, this is not the case. In city we have, apart from buildings, other objects with no preferred alignment, like humans, cars, bicycles, shrubbery, etc. Typically these objects are in front of buildings, thereby generating new spatial frequencies without any orientationpreference by means of occlusion of the background. Because the objects which occlude are typically much smaller than the background-objects (like a man in front of a house), the additionally introduced spatial frequencies should be intermediate to high, and this could help to explain the behavior of the $C O R$ in city and man . A similar explanation can also be employed for natural, and we above described the respective mechanism.

We are now trying to clarify whether the oblique-effect might have its origin in phylogeny or ontogeny. If we take on the phylogenetic point of view as being the origin of the oblique effect (oblique effect due to evolution), then the classes city and man must be excluded from this discussion. The first reason is, of course, that those type of scenes are not natural, because of the presence of manufactured objects. Or, in other words, those scenes occur too infrequently as opposed to natural scenes to provide a representative environment for an evolving organism. The second reason is that the scenes contained in city do not harmonize with what have been found neurophysiologically. To be more precise, city does not show a significant anisotropy at cardinal orientations over the whole range of frequencies (see the corresponding $A E R$-plot figure $5 \mathrm{~b}$ ), although it shows a good alignment at the same time $\left(C O R\right.$-plot figure 5a). As mentioned in the introduction, Coppola et al. ${ }^{7}$ reported that on the average approximately $2 \%$ more cortical area preferred horizontal to vertical orientations. In man we have a significant alignment up to 10 cycles per degree, but no significant alignment beyond this frequency ( $A E R$-plot figure $5 \mathrm{~d})$. 
However, natural shows an corresponding significant anisotropy, with more energy at the vertical orientation at lower spatial frequencies and more energy at horizontal orientation at higher spatial frequencies.

In our point of view it is unlikely that visual experience alone can account to establish the neuronal substrate for the oblique effect. First evidence comes from recent neurophysiological studies. Chapman and Bonhoeffer ${ }^{6}$ examined eight developing and three adult ferrets. In all animals they observed that more cortical area is dedicated to processing of the cardinal orientations. Furthermore, they reported that the individual animals show a great variability in the expression of this effect. This oblique effect could be seen before the onset of the critical period of cortical plasticity, where visual experience gets important. The onset of the critical period in ferrets can be estimated by adding two or three weeks to the beginning of the cat's critical period of susceptibility, which begins at post-natal day 21 (see e.g. ${ }^{20,6}$ and references therein). Furthermore, all ferrets in the Chapman and Bonhoeffer ${ }^{6}$ study were raised in the same visual environment.

But for what reason then does visual experience account? A possible answer to this question is provided by Crair et al. ${ }^{20}$. They studied the impact of visual experience on orientation and ocular dominance maps by comparing normal with binocularly deprived cats. Up to post-natal day 21 the maps of normal and deprived cats were indistinguishable from one another. However, from day 21 on the maps in the binocularly deprived cats begin to deteriorate. Moreover, much stronger and more selective responses were elicited up to post-natal day 21 by stimulation of the contralateral eye compared to the ipsilateral eye. They concluded that pattern vision is required for the maintenance of orientation selectivity rather than for the initial development. Thus, there is evidence that visual experience might serve to establish the responsiveness to the ipsilateral eye in a map which initially is dominated by the contralateral eye. Genes seem to code for an orientation map, and segregation 
of which into ocular dominance columns is accomplished somehow by visual experience.

But do non-optimally driven cells get unresponsive or are they changing their orientation preference? A study of Sengpiel et al. ${ }^{5}$ provides evidence that cells are re-tuned rather than switched off. Moreover, with this result Sengpiel et al. questioned the notion of maintenance of responsiveness. A prerequisite in any case is the plasticity of the underlying neural circuits. And, it would not be surprising if these local cortical networks both mediate ocular-dominance and orientation-selectivity. Hence, one can argue that one cannot switch off the plasticity for orientation-column-circuits while keeping it on for the oculardominance-circuits at the same time, if we assume that they are functionally separated at all. This may be a possible explanation of why visual deprivation in the critical period of susceptibility leads to deterioration of both orientation selectivity and ocular dominance columns. Note that a consequence of taking on this viewpoint is that visual experience can only redistribute poorly used orientation columns rather than creating them from some kind of tabula rasa state.

Let us now reconsider our results and compare the outcomes for the natural sets of images represented by the categories natural, straight, grass and sky. Let us assume that the oblique effect provides an increase in the evolutionary fitness of an organism. We have seen that over the different set of images the expression of the oblique effect differed quite strongly. If we now rely on approximatively one week of visual experience to establish the cortical orientation maps, then we will need to acquire during this time the representative statistics through the set of images perceived. The statistics must be representative in the sense that they are sufficient to establish the oblique effect. For all that this seems unlikely, because during the critical period of cortical plasticity many mammals rely on their parents. Thus, the environment in which they spend the time during the critical period might not have the statistical traits which are needed for an establishment of the oblique effect, although the statistics might be sufficient to establish ocular dominance columns. How far head-rotation 
or other movement induced by active behavior interacts with the establishment of the oblique effect in the critical period, is an issue that has to be more clarified yet. By virtue of our results we can conjecture that this may lead to a decrement of the bigger number of cells at cardinal orientations, i.e. a trend to make the number of cells per orientation equal, so that there in the end the oblique effect cannot longer be observed. So one can conclude by saying that providing orientation maps through genes (including the oblique effect) would be a probably more "secure" way, because the experienced visual statistics in the cortical critical period might not capture always those relevant details which afterwards would provide a potential increase in evolutionary fitness. Note that if this is true, then by not fulfilling the latter requirements, that is solely relying on visual experience, this could amount to an according selection criterion for the evolutionary process, which would possibly converge into the accumulation of the corresponding information into genes (i.e. coding of a single orientation map as a trade off between security and limited space for information storage).

\section{Acknowledgments}

M.K. would like to thank Javier Portilla and Donald Gallogly for fruitful discussions. This work was sponsored by the EU INCO-DC961646 (DG-III) AMOVIP-project, see http://www.iv.optica.csic.es/projects/amovip.html. 


\section{REFERENCES}

1. J.P. Jones and L.A. Palmer, "An evaluation of the two-dimensional Gabor filter model of simple receptive fields in cat striate cortex," J. Neurophysiol.. 58(6) 1233-1258 (1987)

2. S. Apelle, "Perception and discrimination as a function of stimulus orientation: the oblique effect in man and animals," Psychhol. Bull. 78, 226-278 (1972)

3. I.P. Howard, Human Visual Orientation, (Wiley, New York, 1982)

4. B.A. Olshausen and D.J. Field, "Wavelet-like receptive fields emerge from a network that learns sparse codes for natural images," Nature 381, 607-609 (1996)

5. F. Sengpiel, P. Stawinski, and T. Bonhoeffer "Influence of experience on orientation maps in cat visual cortex," Nature Neuroscience 2(8) 727-732 (1999)

6. B. Chapman and T. Bonhoeffer, "Overrepresentation of horizontal and vertical orientations preferences in developing ferret area 17," Proc. Natl. Acad. Sci. USA 95, 2609-2614 (1998)

7. D. Coppola, L. White, D. Fitzpatrick and D. Purves, "Unequal representation of cardinal and oblique contours in ferret visual cortex," Proc. Natl. Acad. Sci. USA 95, $2621-2623(1998)$

8. J.D. Schall, D.J. Vitek and A.G. Leventha, "Retinal constraints on orientation specificity in cat visual cortex," J. Neurosci. 6, 823-836 (1986)

9. D.C. Linden, R.W. Guillery and J. Cucchiaro, "The dorsal lateral geniculate nucleus of the normal ferret and its postnatal development," J. Comp. Neurol. 203, 189-211 $(1981)$

10. M.I. Law, K.R. Zahs and M.P. Stryker, "Organization of the primary visual cortex (area 17) in the ferret," J. Comp. Neurol. 278, 157-180 (1988)

11. E. Switkes, M.J. Mayer and J.A. Sloan, "Spatial frequency analysis of the visual en- 
vironment: anisotropy and the carpentered environment hypothesis," Vis. Res. 18, 1393-1399 (1978)

12. D. Coppola, H. Purves, A. McCoy and D. Purves, "The distribution of oriented contours in the real world," Proc. Natl. Acad. Sci. USA 95, 4002-4006 (1998)

13. A. van der Schaaf and J.H. van Hateren, "Modeling the power spectra of natural images: statistics and information," Vis. Res. 36(17) 2759-2770 (1996)

14. D.J. Field, "Relations between the statistics of natural images and the response properties of cortical cells," J. Opt. Soc. Am. A 12, 2379-2393 (1987)

15. http ://hlab.phys.rug.nl/archive.html

16. F.W. Campbell and D.G. Green, "Optical and retinal factors affecting visual resolution," J. Physiol. 181, 576-593 (1965).

17. J.H. van Hateren and A. van der Schaaf, "Independent component filters of natural images compared with simple cells in primary visual cortex," Proc.R.Soc.Lond. B 265, 359-366 (1998)

18. http://hlab.phys.rug.nl/imlib/peaks.html

19. D.L. Ruderman, "Statistics of natural images: scaling in the woods," Phys. Rev. Let. 73, 814-817 (1994)

20. M.C. Crair, D.C. Gillespie and M.P. Stryker, "The role of visual experience in the development of columns in cat visual cortex," Science 279, 566-570 (1998) 


\section{List of Figures}

1 Left: a single club (representing a weighted integration area in the energy plane) shown at orientation $\phi=0^{\circ}$ (corresponding to vertical image features) and $\phi=90^{\circ}$ (corresponding to horizontal image features). At the right the full "club-swing" in the spatial frequency plane is shown (optical representation). Effective image size is $1023 \times 1023$ pixels. . . . . . . . . . . . . 28

2 The logarithmic average power spectra for each class in a pseudo-color representation (HSV-model). The corresponding classes are from top to bottom and from left to right: city, man, natural, straight, grass, sky, respectively .

3 The oriented energy (OE) as calculated by the CIS using the direct (i.e. original) averaged energy spectrum (ES). The corresponding classes are from top to bottom and from left to right: city, man, natural, straight, grass, sky,

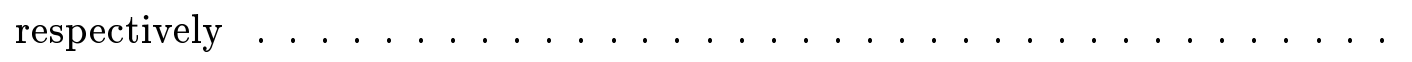

4 Frequency dependence of the oriented energy (OE) calculated from the mean power spectrum (ES) of each class. This was done by using the club integration scheme (CIS) with isotropic blob-like integration areas. The frequency axis was sampled linearly. "Blobs" at different frequencies were simply rescaled versions of each other (with bigger "blobs" at higher spatial frequencies). For reasons of visualization, the energy axis has been rescaled with a factor proportional to the square of the spatial frequency. The corresponding classes are from top to bottom and from left to right: city, man, natural, straight, grass, sky, respectively . . . . . . . . . . 
$5 \quad$ Frequency dependence (part 1) of $C O R$ (left column) and $A E R$ (right column), calculated from the frequency-dependent oriented energy (OE) as shown in figure 4 . Note that the results for natural and straight are summarized in one plot. In the $A E R$-plots, positive values indicate a relative preponderance of vertical image features, while negative values indicate that horizontal image features are dominating at some spatial frequency. The dashed vertical lines refer to the mean value of $<C O R(\sigma=1.5)>$ and $<A E R(\sigma=1.5)>$, respectively, as obtained from uniform distributed noise. The dotted vertical lines are the associated absolute deviations. In the $C O R$ plots the upper dotted vertical line thus corresponds to $C O R_{\text {crit }}$, whereas in the $A E R$-plots the region enclosed between the two dotted vertical lines corresponds to $\triangle A E R_{\text {crit }}$. Note the sign reversal in plot $\mathrm{f}$. Corresponding classes are top row: city, middle row: man, bottom row: natural and straight $\quad$. . 35

6 Frequency dependence (part 2) of the $C O R$ (left column) and $A E R$ (right column). See also the caption of figure 5. Corresponding classes are upper row: grass, lower row: sky . . . . . . . . . . . . . . . . 37

7 Left: cardinal vs. oblique energy ratio $C O R$ calculated from the mean energy spectrum (ES) for each class . Right: the aligned energy ratio $A E R$. Triangles: original ES, squares: $k^{2}$-windowed OE, circles: logarithmic ES. See also the caption of figure $5 . \ldots \ldots \ldots$ 


\section{FIGURES}

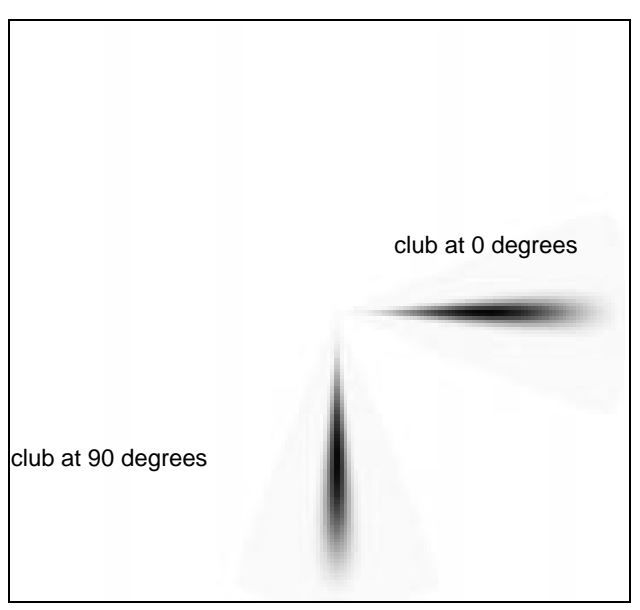

(a)

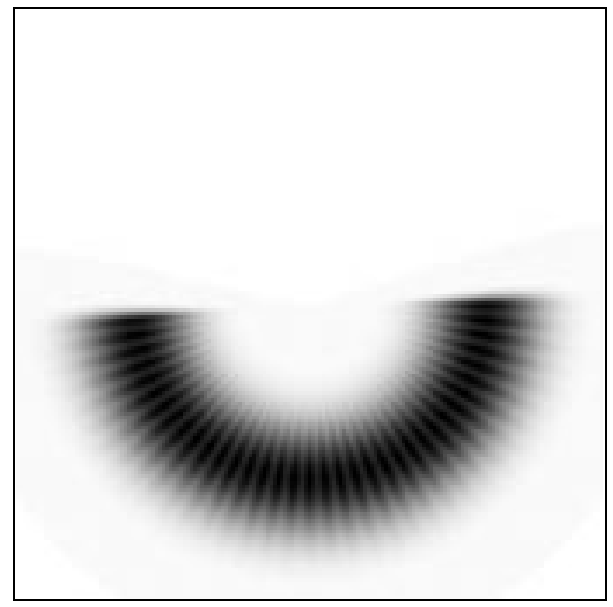

(b)

Fig. 1. Left: a single club (representing a weighted integration area in the energy plane) shown at orientation $\phi=0^{\circ}$ (corresponding to vertical image features) and $\phi=90^{\circ}$ (corresponding to horizontal image features). At the right the full "club-swing" in the spatial frequency plane is shown (optical representation). Effective image size is $1023 \times 1023$ pixels. 


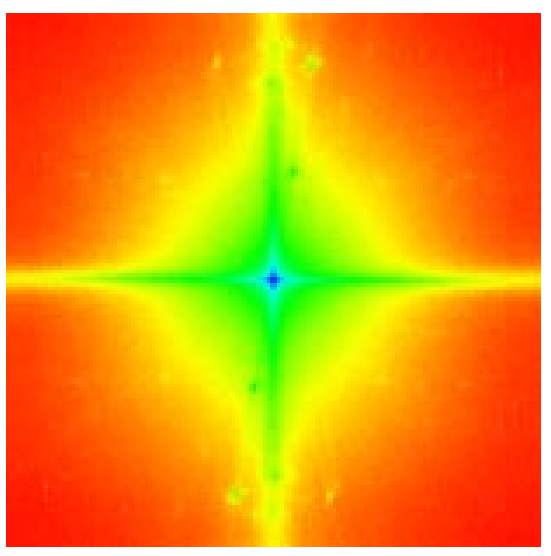

(a)

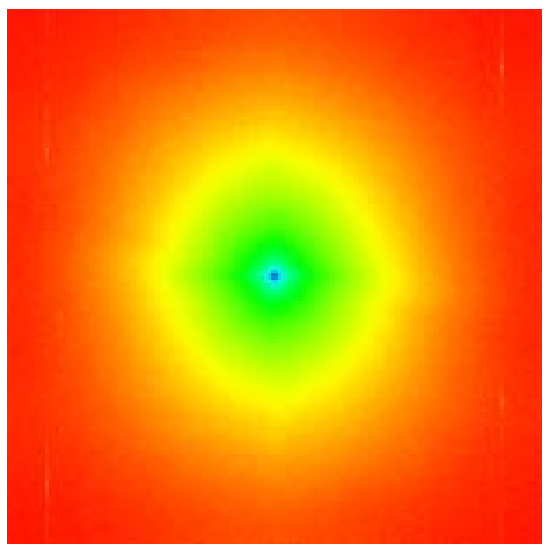

(c)

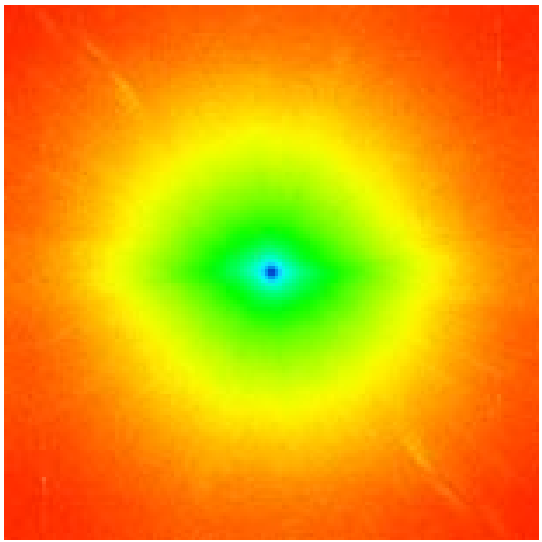

(e)

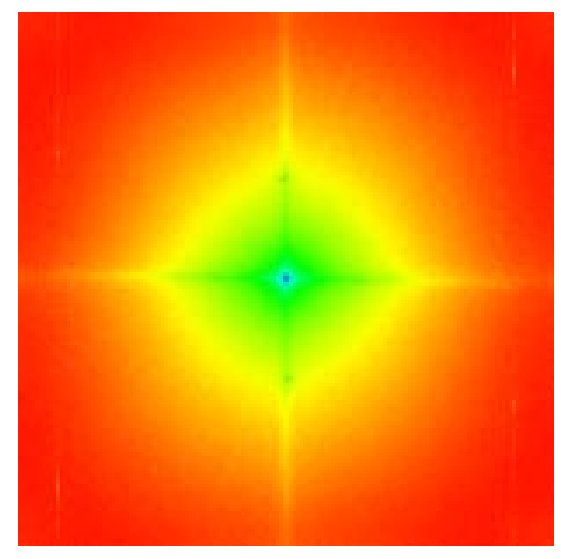

(b)

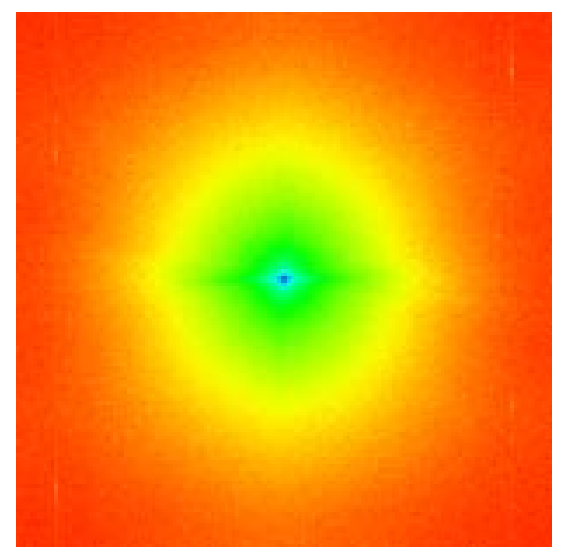

(d)

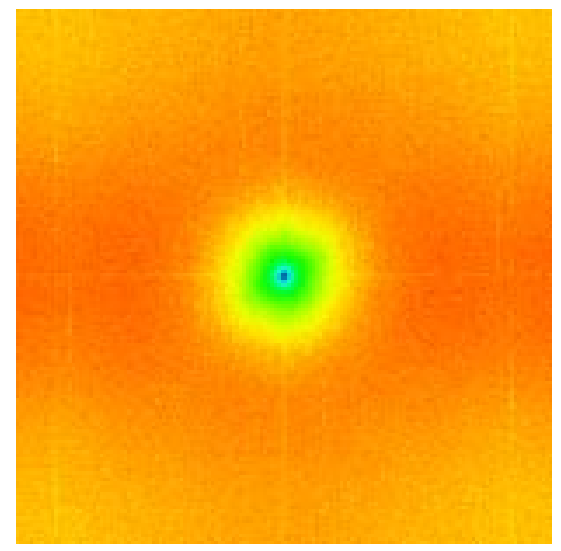

(f) 
Fig. 2. The logarithmic average power spectra for each class in a pseudo-color representation (HSV-model). The corresponding classes are from top to bottom and from left to right: city, man, natural, straight, grass, sky, respectively 
Oriented energy $E_{\alpha}(\phi)$ for "city", $\operatorname{COR}(\sigma=1.5)=0.89467$

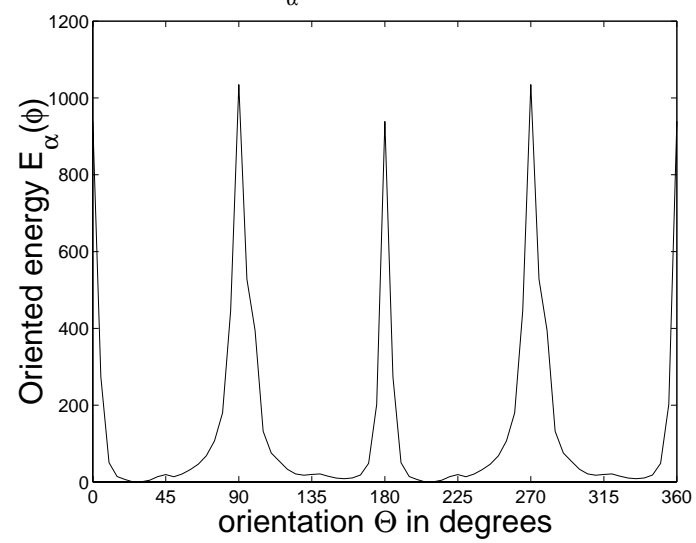

(a)

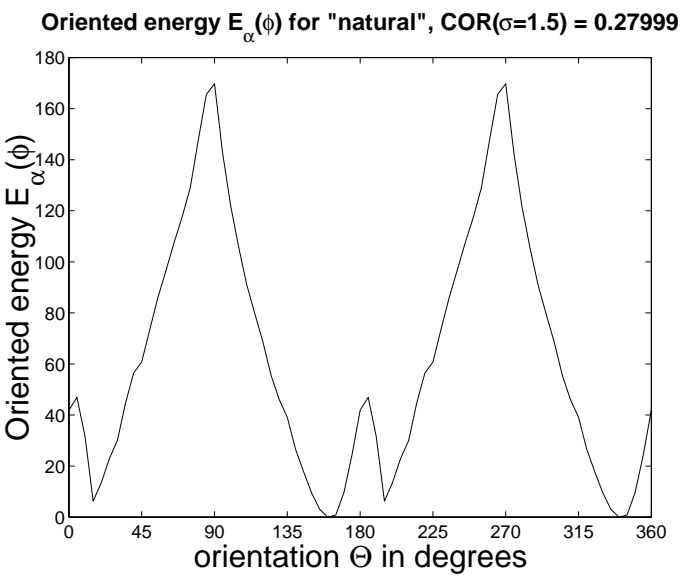

(c)

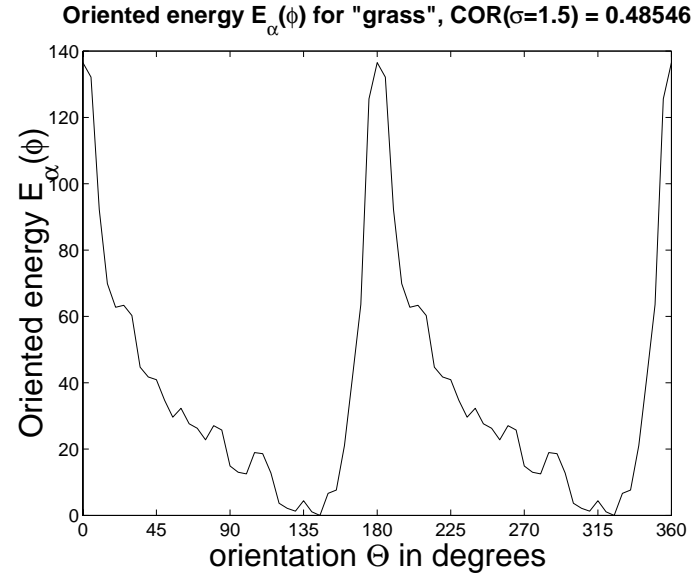

Oriented energy $E_{\alpha}(\phi)$ for "man", $\operatorname{COR}(\sigma=1.5)=0.70712$

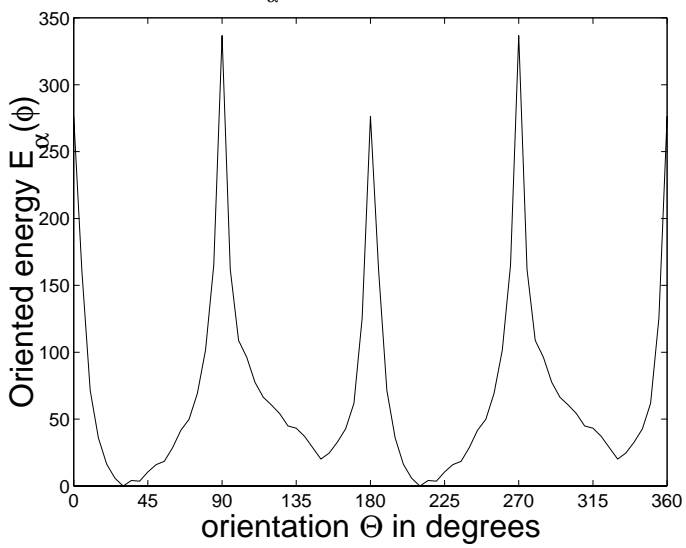

(b)

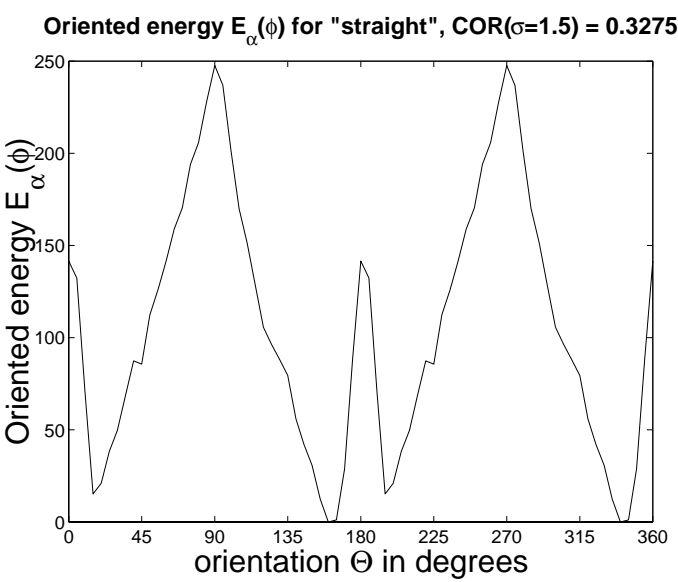

(d)

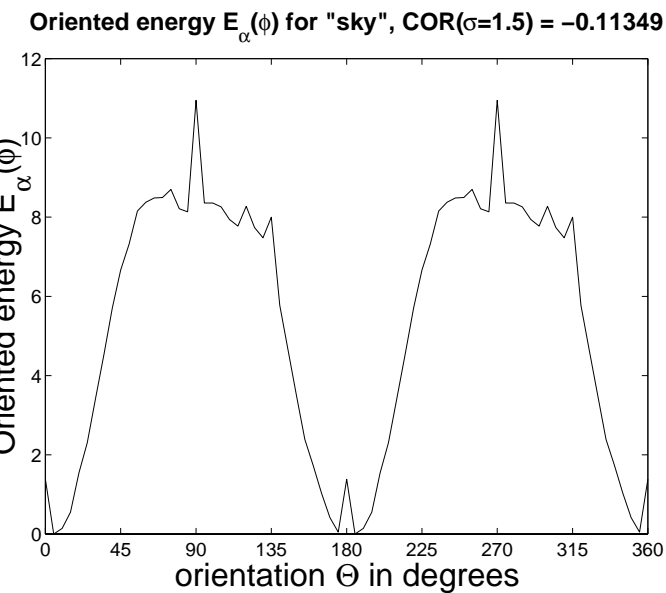


Fig. 3. The oriented energy (OE) as calculated by the CIS using the direct (i.e. original) averaged energy spectrum (ES). The corresponding classes are from top to bottom and from left to right: city, man, natural, straight, grass, sky, respectively 
Oriented energy vs. spatial frequency for "city"

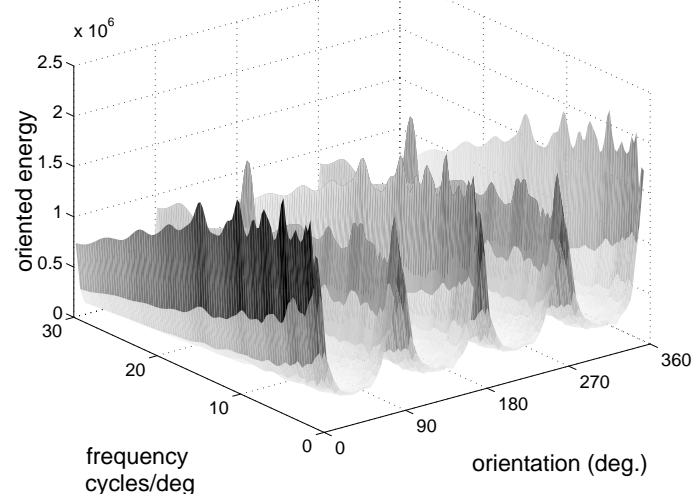

(a)

\section{Oriented energy vs. spatial frequency for "natural"}

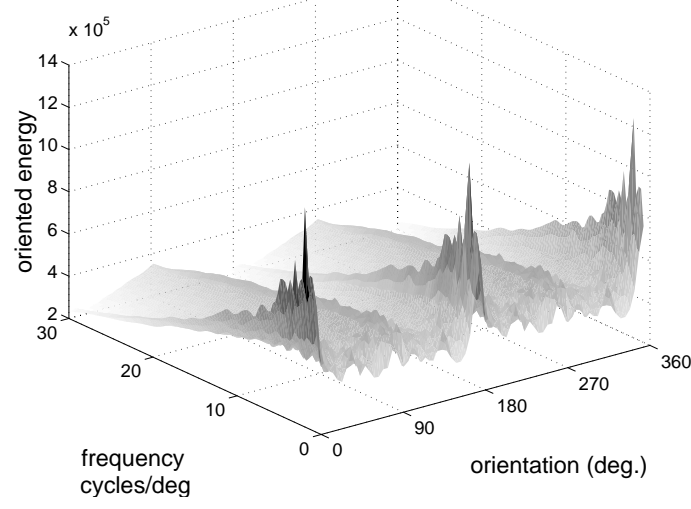

(c)

Oriented energy vs. spatial frequency for "grass"

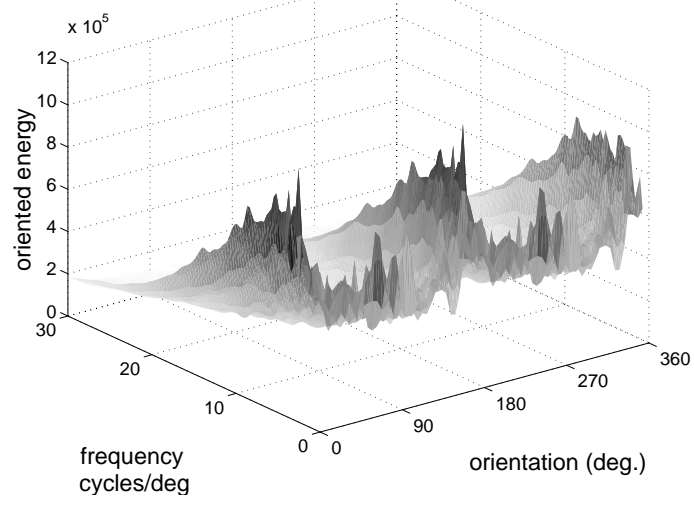

(e)
Oriented energy vs. spatial frequency for "man"

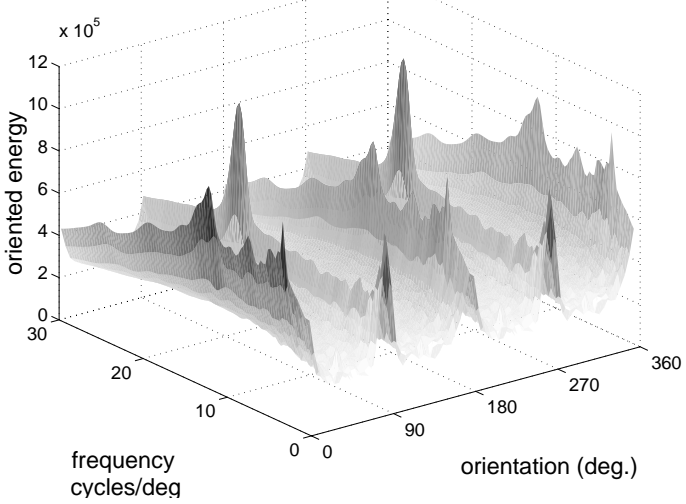

(b)

\section{Oriented energy vs. spatial frequency for "straight"}

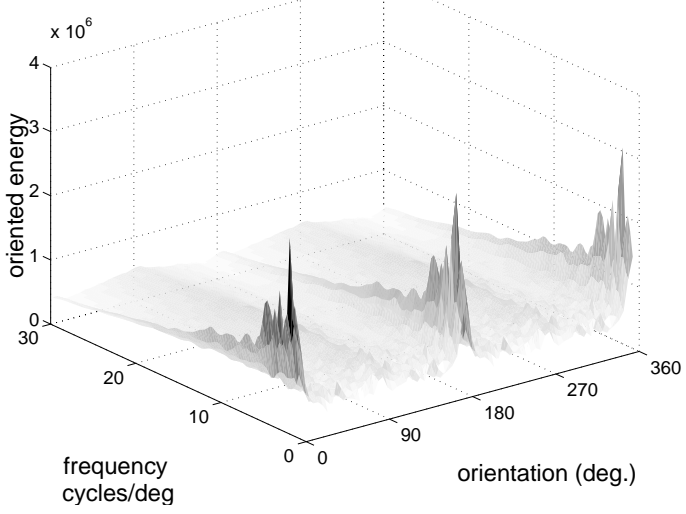

(d)

Oriented energy vs. spatial frequency for "sky"

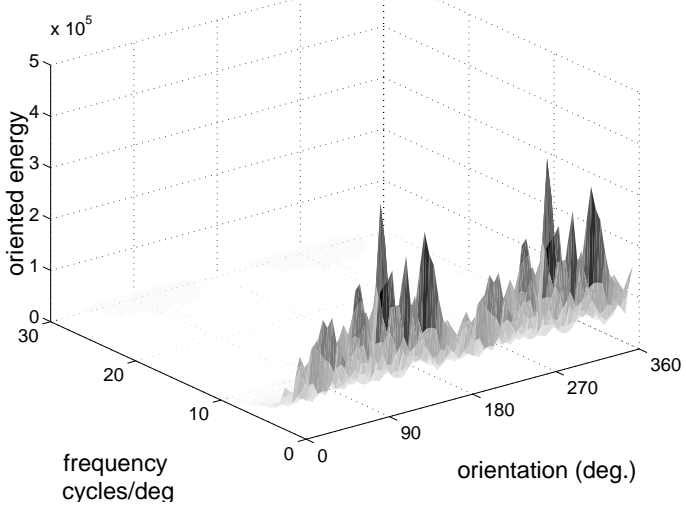

(f) 
Fig. 4. Frequency dependence of the oriented energy (OE) calculated from the mean power spectrum (ES) of each class. This was done by using the club integration scheme (CIS) with isotropic blob-like integration areas. The frequency axis was sampled linearly. "Blobs" at different frequencies were simply rescaled versions of each other (with bigger "blobs" at higher spatial frequencies). For reasons of visualization, the energy axis has been rescaled with a factor proportional to the square of the spatial frequency. The corresponding classes are from top to bottom and from left to right: city, man, natural, straight, grass, sky, respectively 


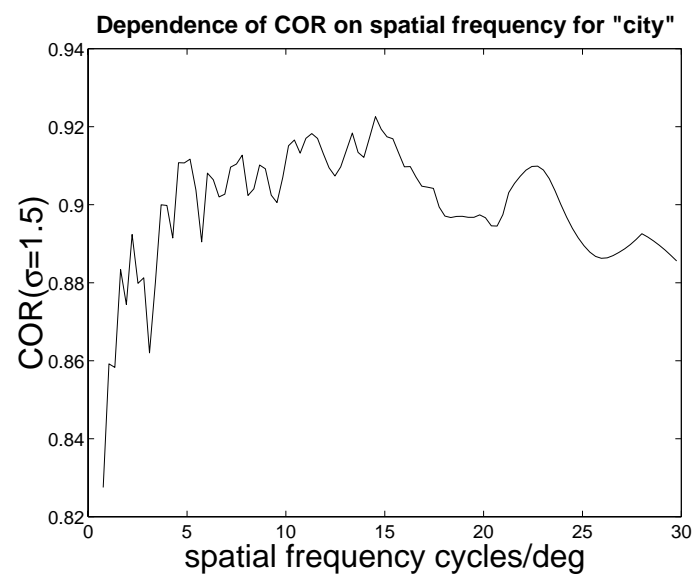

(a)

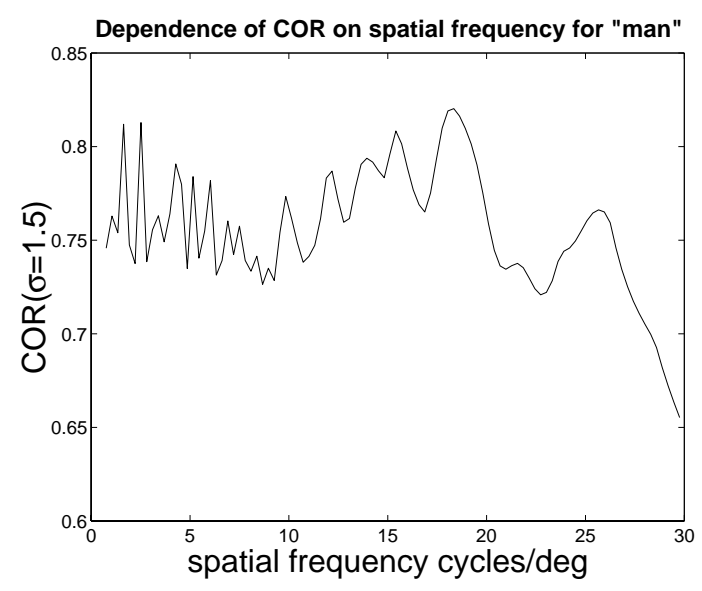

(c)

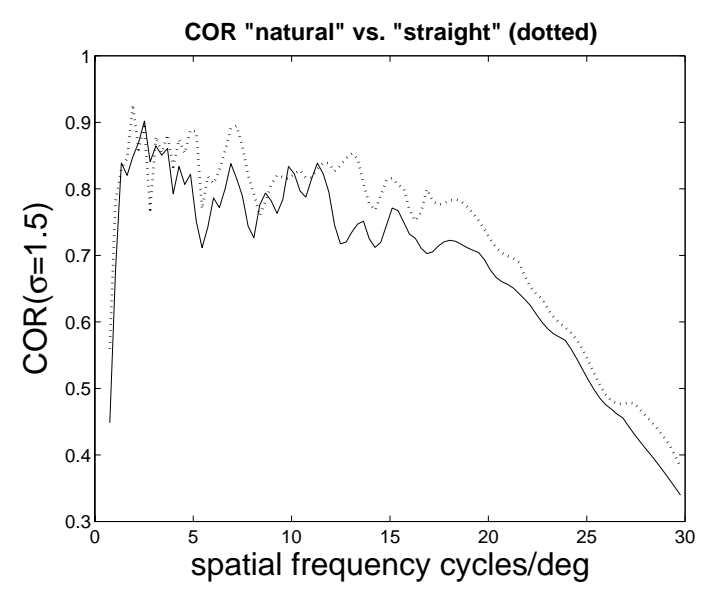

(e)

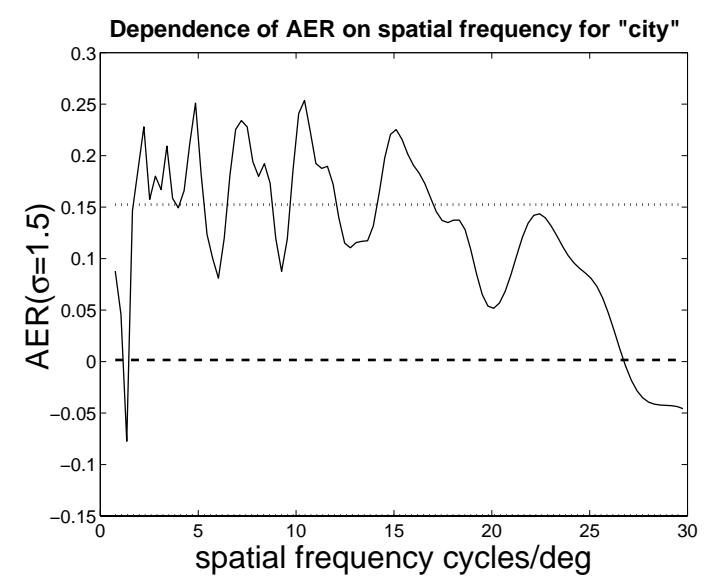

(b)

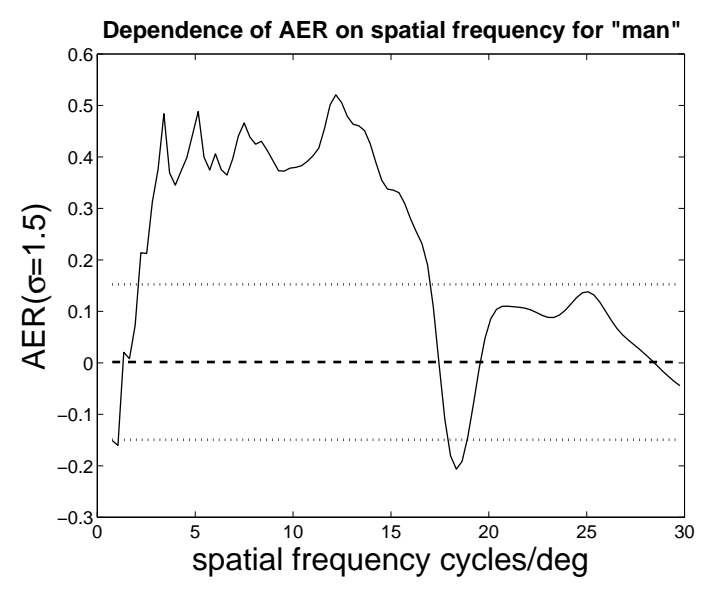

(d)

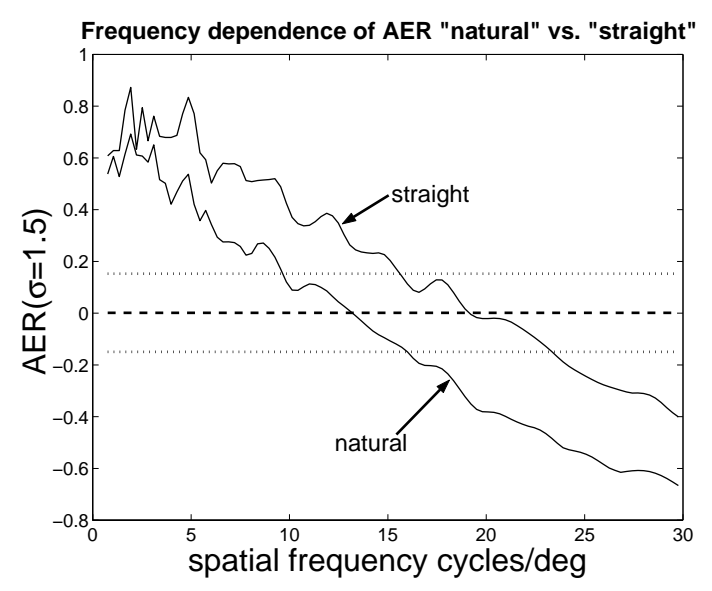

(f) 
Fig. 5. Frequency dependence (part 1) of $C O R$ (left column) and $A E R$ (right column), calculated from the frequency-dependent oriented energy (OE) as shown in figure 4. Note that the results for natural and straight are summarized in one plot. In the $A E R$-plots, positive values indicate a relative preponderance of vertical image features, while negative values indicate that horizontal image features are dominating at some spatial frequency. The dashed vertical lines refer to the mean value of $\langle\operatorname{COR}(\sigma=1.5)>$ and $\langle A E R(\sigma=1.5)>$, respectively, as obtained from uniform distributed noise. The dotted vertical lines are the associated absolute deviations. In the $C O R$-plots the upper dotted vertical line thus corresponds to $C O R_{\text {crit }}$, whereas in the $A E R$-plots the region enclosed between the two dotted vertical lines corresponds to $\triangle A E R_{\text {crit }}$. Note the sign reversal in plot $\mathrm{f}$. Corresponding classes are top row: city, middle row: man, bottom row: natural and straight 


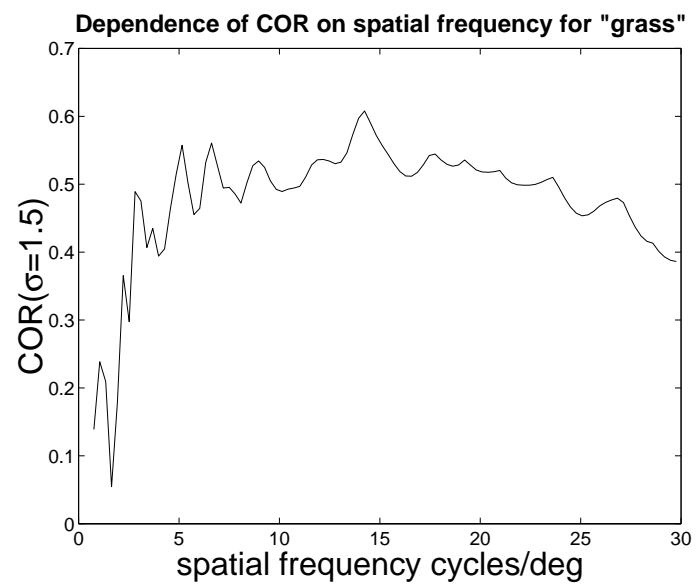

(a)

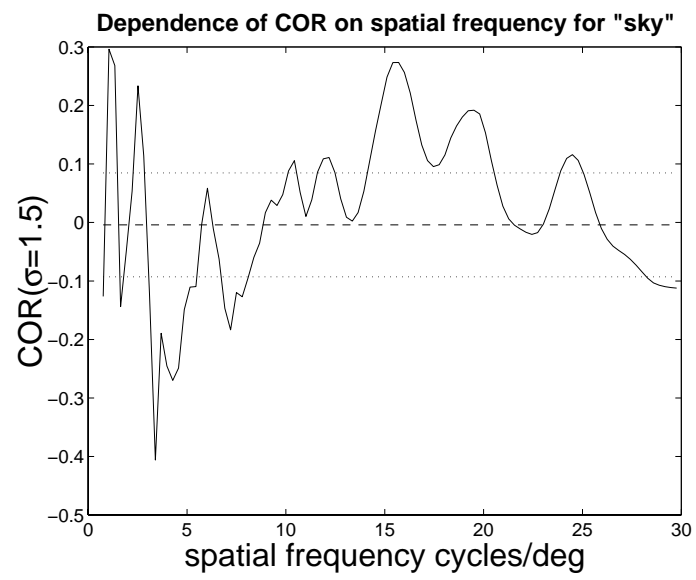

(c)

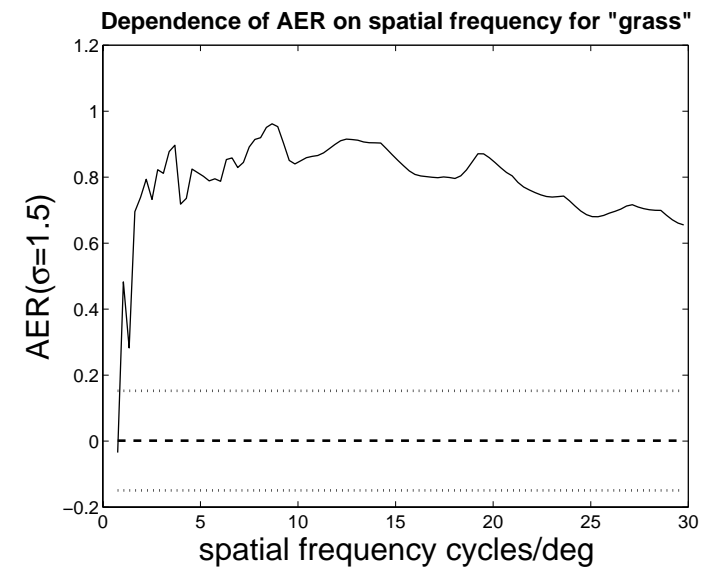

(b)

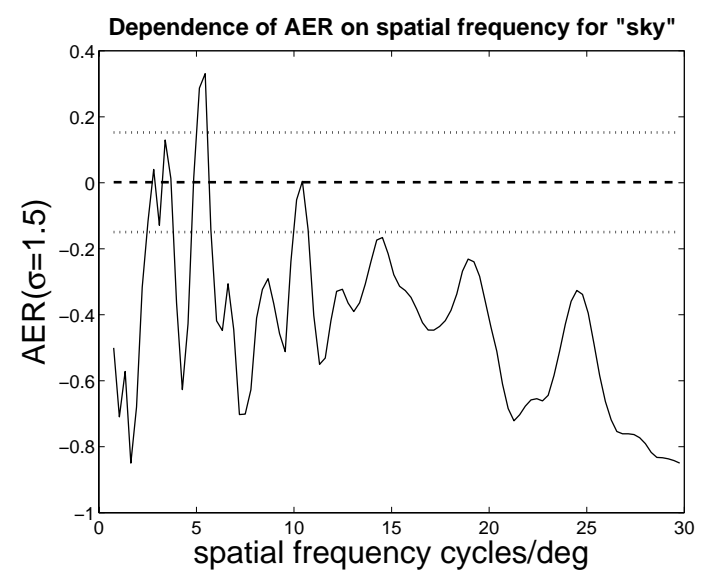

(d)

Fig. 6. Frequency dependence (part 2) of the COR (left column) and $A E R$ (right column). See also the caption of figure 5. Corresponding classes are upper row: grass, lower row: sky 


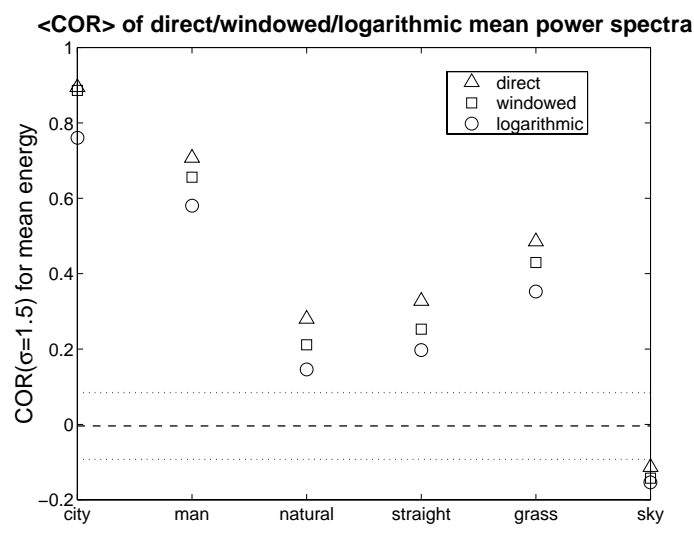

(a)

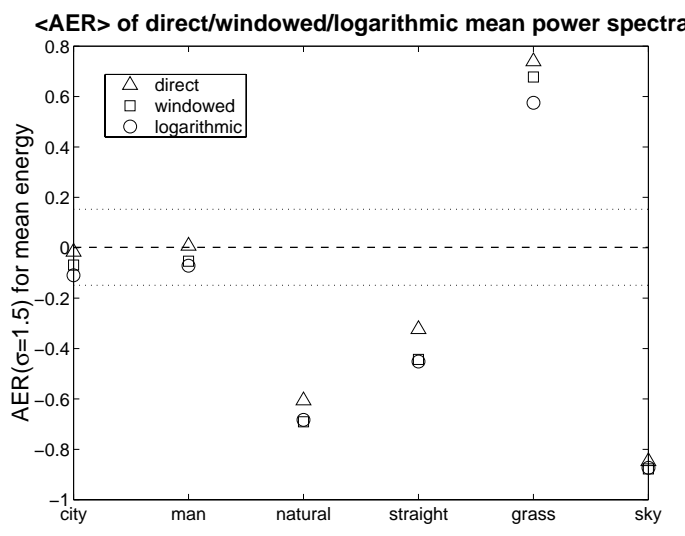

(b)

Fig. 7. Left: cardinal vs. oblique energy ratio $C O R$ calculated from the mean energy spectrum (ES) for each class . Right: the aligned energy ratio $A E R$. Triangles: original ES, squares: $k^{2}$-windowed OE, circles: logarithmic ES. See also the caption of figure 5 . 


\section{List of Tables}

1 Here the number of images is shown fulfilling $C O R \notin \Delta C O R_{\text {crit }}$ while using the raw ES ("direct"), the $k^{2}$-windowed ES and the logarithmic ES. The column "total" refers to the number of images in each class. . . . . . . . . . 40

2 Here the number of images is shown fulfilling $A E R \notin \Delta A E R_{\text {crit }}$ while using the unmodified ES ("direct"), the $k^{2}$-windowed ES and the logarithmic ES. The column "total" refers to the number of images in each class. . . . . . . 


\section{TABLES}

\begin{tabular}{|c||c|c|c|c|}
\hline \hline Short-cut & total & direct & $k^{2}$-windowed & logarithmic \\
\hline \hline city & 121 & $119(98.3 \%)$ & $116(95.9 \%)$ & $110(90.9 \%)$ \\
\hline man & 179 & $137(72.9 \%)$ & $128(68.1 \%)$ & $106(56.4 \%)$ \\
\hline natural & 275 & $90(37.3 \%)$ & $74(30.7 \%)$ & $43(17.8 \%)$ \\
\hline straight & 56 & $28(50.0 \%)$ & $23(41.1 \%)$ & $16(28.6 \%)$ \\
\hline grass & 41 & $27(55.1 \%)$ & $26(53.1 \%)$ & $25(51.0 \%)$ \\
\hline sky & 9 & none & none & none \\
\hline
\end{tabular}

Table 1. Here the number of images is shown fulfilling $C O R \notin \Delta C O R_{\text {crit }}$ while using the raw ES ("direct"), the $k^{2}$-windowed ES and the logarithmic ES. The column "total" refers to the number of images in each class. 


\begin{tabular}{|c||c|c|c|c|}
\hline \hline Short-cut & total & direct & $k^{2}$-windowed & logarithmic \\
\hline \hline city & 121 & $87(71.9 \%)$ & $85(70.2 \%)$ & $59(48.8 \%)$ \\
\hline man & 179 & $164(87.2 \%)$ & $165(87.8 \%)$ & $162(86.2 \%)$ \\
\hline natural & 275 & $224(92.9 \%)$ & $228(94.6 \%)$ & $221(91.7 \%)$ \\
\hline straight & 56 & $52(92.9 \%)$ & $53(94.6 \%)$ & $54(96.4 \%)$ \\
\hline grass & 41 & $45(91.8 \%)$ & $45(91.8 \%)$ & $45(91.8 \%)$ \\
\hline sky & 9 & $9(100 \%)$ & $9(100 \%)$ & $9(100 \%)$ \\
\hline
\end{tabular}

Table 2. Here the number of images is shown fulfilling $A E R \notin \Delta A E R_{\text {crit }}$ while using the unmodified ES ("direct"), the $k^{2}$-windowed ES and the logarithmic ES. The column "total" refers to the number of images in each class. 